\title{
Recenti risultati sulla velocità delle onde sismiche in Italia Centrale
}

(Some notes on the velocity of seismic-waves in Central Italy)

\author{
R. Coxsole (*) - C. Gasparixi (*)
}

Rirevulo il 20 . Marzo 1975

\begin{abstract}
Rrasicsto.... Il periodo sismico di ('erreto di spoleto (Diembre 1974) è stato studiato con i dati ottenuti dalle stazioni della Rete Nazionale e dallat stazione sismica mobile, posta in prossimit à dellepicentro. Grazie alla possibilita di disporre di una buona localizzazione degli ipocentri e dei tempi di origine assoluti, si sono determinate le dromocene medie relative alle onde $P \prime g, S q, P n$ e $S ' n$. Por brevi distanze di tragitto $\dot{s}$ stato possibile mettere in evidenza forti anomalie azimutali nelle velocita delle onde sismiche, associabili a crori sistematici nella determinazione decrli epicentri con $i$ metodi classiei. Il presente studio fa prevedere la possibilita di giungere ad una conoseenza piu dettagliata delle caratteristiche elastiche della crosta in territorio nazionale, quale risultato del perfezionamento teenieo nella raceolta dei dati.
\end{abstract}

SUMnarr. - The seismie phenomena which happened near cerreto di spoleto (1)ecember 1974) have been st udied hy data collected from the National letwork and the seismic mobile slation. The latter had been placed in the epicentral zone. As we conld know good hypoecentral parameters, we determined travel times for $P q, S q, P n$ and $S^{\prime} n$ waves. For short travel distances it has been possible to point ont strong azimnthal velocity anomalies, connected with systematie errors in the epicentral delemination. This work allows us to foresee the possibility of improving the knowledge of the crustal characteristies in our comntry, as far as recording techniques will improve.

(*) Istituto Nazionale di Goofisica, Roma. 


\section{1. - PREMESSI}

Nella prima metà di Dicembre $197 \mathrm{f}$ si è manifestato, nella Valle del Nera, un periodo sismico caratterizzato da cirea 20 scosse di magnitudo locale superiore a 3.0 e da molte altre di magnitudo inferiore. Ia scossa più forte è stata quella del giorno 2 Dicembre alle $01155^{\mathrm{m}} 15.2^{\mathrm{s}}$ avente magnitudo $M_{\mathrm{L}}$ pari a 4.3 , determinata con il Wood-Anderson dell'Osservatorio di Monte Porzio, alla distanza di eirea $115 \mathrm{~km}$.

Il periorlo sismico i stato seguito con numerose registrazioni clalle stazioni della Rete Nazionale, delle quali cinque (e precisamente Perugia, I'Aquila, Tuscania, Corinaldo, Roma M.P.) erano a distanza utile per ricevere l'onda $P g$ come primo arrivo (fig. 1).

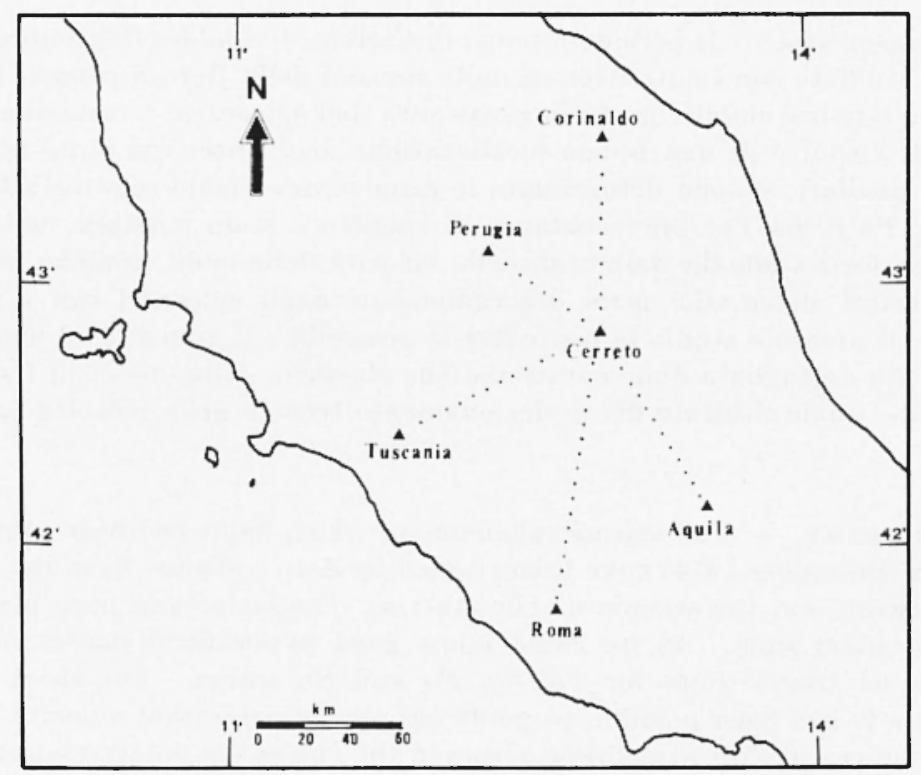

Fig. 1 - Posizione delle stazioni prossime allepiecntro.

A partire dalla sera del 7 Dicembre è entrata in funzione, nell'abitato di C'erreto di Spoleto, la stazione mobile dell'Istituto Nazionale di Ceofisica dotata anche di registratore magnetico. La stazione mobile, 
trovandosi praticamente sullepicentro dei sismi, ha permesso la localizzazione dei fuochi ( $\left.{ }^{2}\right)$ i quali sono risultati tutti in un'area di alcuni chilometri quadrati ad est di Cerreto, centrata approssimativamente sul punto di coorlinate $42^{\circ} 49^{\prime} \mathrm{N}$ e $12{ }^{\circ} 56^{\prime} \mathrm{E}$, ed alla profondita compresa tra due e quattro chilometri (fig. 2). Il metorlo aroperato per la localizad-

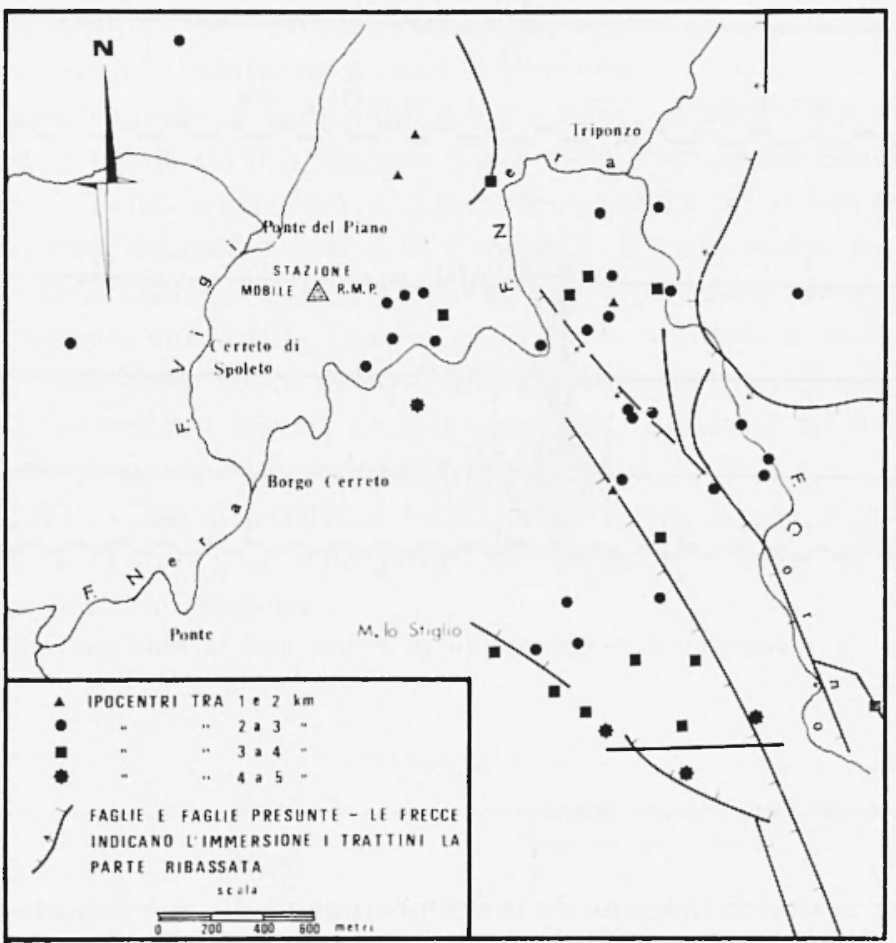

Fig. 2 - Epicentri determinati con la stazione mobile dal griorno $i$ al 13 dicembre.

zione degli epicentri ì stato naturalmente quello rlelle distanze ipocentrali e dell'angolo di emergenza, ottenuti dalle registrazioni della terna di sismografi della stazione mobile (fig. 3). Un altro risultato consentito dall'impiego della stazione mobile, come vedremo in seguito, is stato quello di individuare con esattezza il tempo origine di alcune scosse. 

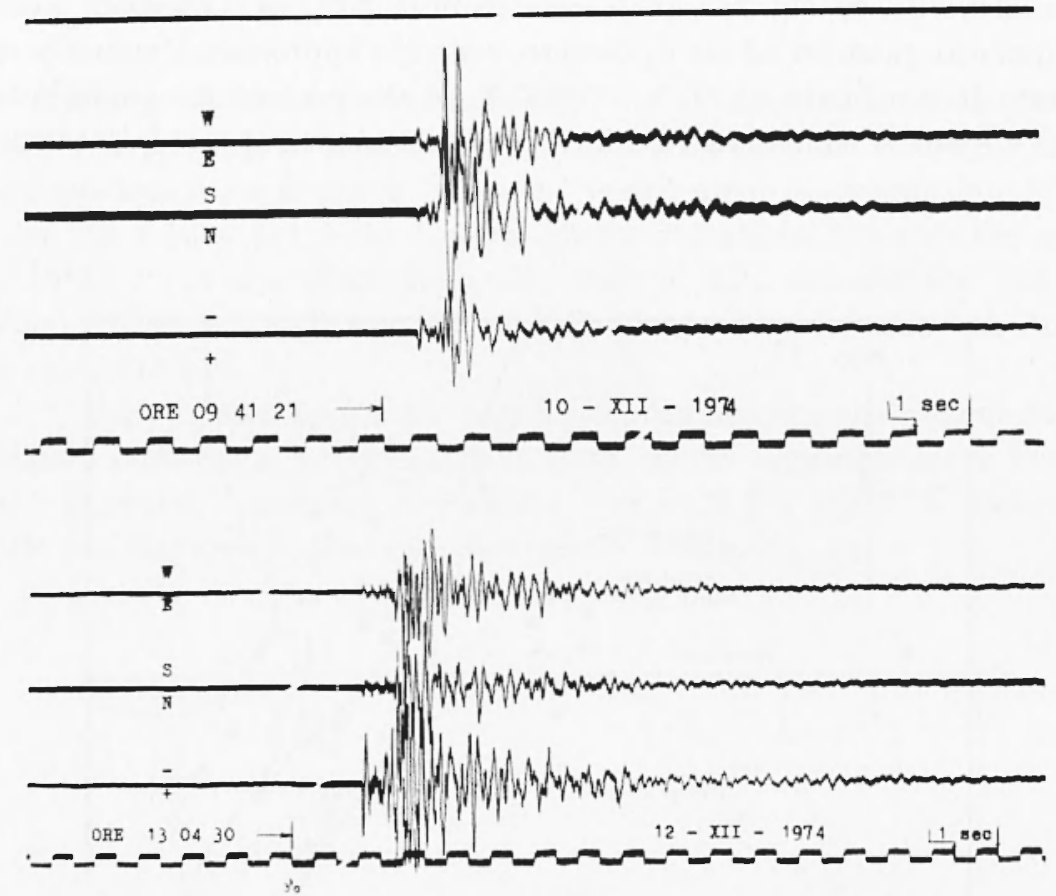

Figr. 3 - Esempi di registrazioni oftenute con la stazione mobile.

\section{2. - IJE DROMOCRONE MEDIE}

Per la determinazione delle dromocrone medie si è assunta l'ipotesi che, anche le soosse avvenute nei giorni precedenti alla messa in opera della stazione mobile, abbiano avuto la loro origine nel volume di roceia interessato dalle repliche registrate in seguito (1,5). Questa ipotesi a sembra attendibile per il fatto, generalmente ammesso, che le repliche di un sisma avvengano nella zona posta in squilibrio dalla variazione di tensione elastica introdotta dalla scossa principale, el i confortata dall'uniformità dei tempi di tragitto ricavati per le varie stazioni nel rorso del periodo sismico.

Le repliche da noi localizzate si distribuiscono su di monea di rirca $10 \mathrm{~km}^{2}$. Ponendoci nella peggione delle ipotesi, a cioc che le scosse utilizate nella trattazione si localizzino al bordo dell'area, lerrore che 
commetteremo nell'assumere come epicentro il centro dell'area ileterminata dalle repliche (fig. 2), sarà valutabile nei seguenti modi:

a) considerando larea come circolare con ragrgio di circa $1.8 \mathrm{~km}$ a assumendo la velocita di $5.5 \mathrm{~km} / \mathrm{sec}$, commetteremo un errore di 0.3 secondi;

b) considerando invece l'area quarlrata, il massimo spostamento ì li $2.2 \mathrm{~km}$, con un errore corrispondente a 0.4 secondi.

In ambelue i casi l'errore commesso rientra in quello di lettura sui sismogrammi, valutabile a circa 0.5 secondi.

Successivamente si sono confrontati i tempi di arrivo alla stazione sismica dell'Aquila (WWNSS), con quelli della stazione mobile corretti in funzione della brevissima distanza ipocentrale. Si i ottenuto un tempo di tragitto melio pari a 10.8 secondi. E stata scelta come stazione di riferimento quella dell' $A$ quila sia per la posizione e la sensibilità legli strumenti che per la minore dispersione nei dati ottenuti. Tale tempo ì stato preso come lato di riferimento per valutare lora origine clelle precedenti scosse, da impiegare nel calcolo di tutti i tempi di tragitto verso le altre stazioni.

Stabiliti, come si ì detto, i tempi origine delle scosse di maggiore importanza (Tab. 1), si sono potuti determinare i tempi di tragritto relativi a tutte le stazioni.

T'ABEIAA I

\begin{tabular}{|c|c|c|c|c|c|c|}
\hline So & \multicolumn{2}{|c|}{ Tata } & \multicolumn{3}{|c|}{$\begin{array}{l}\text { Tempo origrime } \\
\mathrm{h}_{\mathrm{S}} \mathrm{m}\end{array}$} & $M_{L H}$ \\
\hline 1 & \multicolumn{2}{|c|}{ 1.X11.1974 } & (1) & 21 & 28.9 & 3.9 \\
\hline 2 & 2 & $"$ & 01 & 14 & 32.7 & 3.8 \\
\hline 3 & 2 & $"$ & 01 & $\overline{5} \overline{5}$ & 15.2 & 4.3 \\
\hline 4 & 2 & $"$ & 02 & 46 & 32.5 & 3.3 \\
\hline 5 & $\underline{2}$ & $"$ & 21 & 00 & 11.2 & 3.7 \\
\hline 6 & 3 & $"$ & 19 & 118 & 12.4 & 3.6 \\
\hline 7 & 4 & $"$ & (1) & 39 & 37.7 & 3.2 \\
\hline 8 & 4 & $"$ & 16 & 14 & 48.2 & 4.0 \\
\hline 9 & 4 & $”$ & 17 & 59 & $4 \overline{5} . \overline{5}$ & 3.6 \\
\hline 10 & 5 & $"$ & 0.5 & 22 & 0.5 .4 & 4.1 \\
\hline 11 & 5 & $"$ & $0 \overline{5}$ & 45 & 49.1 & 3.4 \\
\hline 12 & 6i & $n$ & 17 & 49 & 02.6 & 3.7 \\
\hline
\end{tabular}


Tel fare ciò abbiamo distinto i quattro tipi fondamentali di oncle $P y, P n, s g, \$ n$, i cui nomi sono da consiclerarsi puramente convenzionali, ricavando i rispettivi tempi ogni volta che l'arrivo si poteva distinguere con certezza. Si noti che per le cinque stazioni più vicine si sono letti direttamente i sismogrammi, mentre per le altre ci si à basati sui dati indicati nei bollettini.

T risultati per le onde $P y$ e $S y$ sono riportati nelle Tabelle 2 a 3 dove, accanto al nome della stazione, abbiamo indicato la distanza

TABLLA 2

\begin{tabular}{|c|c|c|c|c|}
\hline Stazione & $\begin{array}{c}1 \\
(k+11)\end{array}$ & $\begin{array}{c}T_{P_{0}} \\
(\mathrm{sec})\end{array}$ & $\begin{array}{c}V_{l^{\prime} g} \\
\left(\mathrm{~km} / \mathrm{sec}^{\prime}\right)\end{array}$ & $x$ \\
\hline I'ERUGia & 53.8 & $11.15 \pm 0.22$ & $4.82+0.09$ & 12 \\
\hline IAAQUHA & 64.2 & 10.80 & 5.94 & - \\
\hline ('orixatoo) & 90.6 & $17.80+0.10$ & $5.09 \pm 0.03$ & 14 \\
\hline Tuscania & 97.2 & $16.74+0.08$ & $5.81 \pm 0.02$ & 7 \\
\hline Rowa M.P'. & 113.4 & $19.37 \pm 0.13$ & $5.85 \pm 0.04$ & 11 \\
\hline FIRENZE & 172.6 & $31.01 \div 0.42$ & $5.57 \pm 0.07$ & 7 \\
\hline P'RATO & 189.8 & $33.11=0.39$ & $5.73 \div 0.060$ & 7 \\
\hline
\end{tabular}

epicentrale $A$ - ealcolata trigonometricamente clal punto inclicato in premessa - il tempo di tragitto medio e le velocitì risultanti con i loro errori stancard, nonché il numero $N$ a osservazioni impiegate nella determinazione.

TABRLLA 3

\begin{tabular}{|c|c|c|c|c|}
\hline Stazione & $\stackrel{1}{(k+11)}$ & $\begin{array}{l}T_{S g} \\
\left(\mathrm{sec}^{\prime}\right)\end{array}$ & $\begin{array}{c}Y_{S g} \\
(\mathrm{~km} / \mathrm{sec})\end{array}$ & N \\
\hline P'ERUGIA & 53.8 & $20.85=0.40$ & 2.58 上 0.05 & 12 \\
\hline I:AQUUA & 64.2 & $20.38 \pm 0.22$ & $3.15+0.03$ & 13 \\
\hline (0)RINAl,o) & 90.6 & $31.59 \pm 0.15$ & $2.87 \div 0.01$ & 14 \\
\hline Tuscania & 97.2 & $32.37 \pm 0.27$ & $3.00 \pm 0.02$ & 8 \\
\hline ROMA M. I'. & 113.4 & $36.60 \pm 0.14$ & $3.10 \pm 0.01$ & 12 \\
\hline FIRENZE & 172.6 & $55.68 \div 0.60$ & $3.10=0.03$ & 7 \\
\hline P'RATO & 189.8 & $61.92 \pm 1.12$ & $3.07 ! 0.06$ & 4 \\
\hline
\end{tabular}


In Tabella 1 sono riportati i risultati per le onde $P$ e s'n, lovo naturalmente non sono state indicate le velocita medie, trattanciosi di pereorsi non rettilinei.

'TABELAS 4

\begin{tabular}{|c|c|c|c|}
\hline Stazione & $\begin{array}{c}4 \\
(\mathrm{k} m)\end{array}$ & $T_{P n}$ & $T_{S n}$ \\
\hline PRATO & 189.8 & - & 54.39 \\
\hline (ENOYA & 368.8 & 53.20 & $\ldots$ \\
\hline Pavia & 399.9 & 60.30 & - \\
\hline ROBURENT & $4+1) .6$ & 63.35 & - \\
\hline SARAIEYO & $460.6 j$ & 66.80 & 120.70 \\
\hline S. ANNA DI $V$. & 480.1 & 67.80 & $\ldots$ \\
\hline 'TITOCRAL) & 520.5 & 73.98 & 128.23 \\
\hline ()IIRII) & 678.4 & 92.72 & - \\
\hline SCOPJE & 706.1 & 95.84 & - \\
\hline VAlaxiogo & 813.9 & 109.76 & - \\
\hline I)EVA & 863.3 & 117.80 & - \\
\hline Moxa & 875.9 & 119.08 & - \\
\hline
\end{tabular}

A partire dai tempi e dalle distanze epicentrali liportati nelle tabelle si sono calcolati, col metodo dei minimi qualrati, i coefficienti delle equazioni delle dromocrone, assunte come rette. I risultati ottenuti, dei quali i primi due rivestono maggior signifiato per il numero dei dati a disposizione, sono i seguenti:

$$
\begin{array}{ll}
\text { onda } P y & T_{P g}=(0.171 \pm 0.006) A+0.862 \pm 0.700 \\
\text { onda sy } & T_{s_{\eta}}=(0.31+ \pm 0.008) A+1.893 \pm 0.897 \\
\text { oncla } P_{n} & T_{P_{n}}=(0.126 \pm 0.002) A+7.999 \pm 1.122 \\
\text { onda sn } & T_{s n}=(0.230 \pm 0.018) A+11.376 \pm 7.476
\end{array}
$$

dove i tempi sono espressi in secondi e le distanze in $\mathrm{km}$.

Queste dromocrone sono valide per terremoti con profonditi ipocentrale di circa $4 \mathrm{~km}$, e sono molto simili a quelle ricavate da Di Filippo e Marcelli $\left(^{4}\right)$. Va, commuque, notato che in questa circostanza si sono aruti a disposizione piu dati per le brevi distanze. 


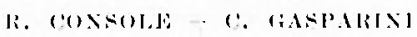

\section{3. - ('RITICA MAL DETERMINAZIONE CLASSICA DEGLI EPICENTRI}

E stata tentata la determinazione degli epicentri per le soosse più significative con clue fra i metodi più classici: quello che si basa sui soli primi arrivi e quello basato sulle differenze $T_{s q}-T_{r q}$. Per il primo, che comporta fra le incognite anche il tempo origine, si somo anoperati tutti i dati a disposizione assumenclo come valiale le dromocone determinate da Di Filippo e Marcelli $\left(^{-1}\right)$. Per il secondo abbiamo considerato incognita la costante $l_{i}=-\frac{Y_{P q} \cdot V_{s g}}{V_{r g}-V_{s g}}$ fissando invece come lata la profonditi ipocentrale. Ambedue i metorli sono stati tralotti in un programma di minimi qualrati per l'elaboratore elettronico.

I risulati ottenuti, simboli neri di fig. 1, somo stati piuttosto deludenti in quanto presentano una forte dispersione, dipendente dagli errori casuali nella lettura dei tempi di arrivo delle onde, e sono anche affetti da errori sistematici a causa delle differenze ta le velociti reali delle onde e quelle assunte nel modello. Lo spostamento verso sud di tutti gli epicentri determinati col metodo delle $P$, per esempio, può provenire dal fatto che la velocita delle onde sismiche s maggiore in tale direzione, come ì stato confermato dall'analisi che segue.

A scopo di verifica sono stati ricalcolati gli epicentri con il metodo delle $I$, utilizando per ogni stazione le velocita riportate in Tabella 2 (triangoli bianchi, figre. 4 e 5 ).

Un'altra differenza che si o rilevata fra i dati ottenuti dal calcolo e gli stessi dati ricavati direttamente, sta nei tempi origine, per $i$ quali i valori forniti dal metodo classico sono sistematicamente inferiori di alemini secondi.

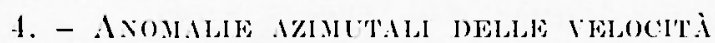

Dallesame dei risultati riportati nelle Tabelle 2 e 3 si puo osservare ma notevole differenza nelle velocità delle onde in relazione al particolare tragitto. E importante far notare che ciò deriva dall'aver determinato per via diretta la posizione epicentrale e il tempo origine dei terremoti, eliminando l'inevitabile "aggiustamento" che deriverebbe dalladoperare dromocrone medie per determinare i para- 


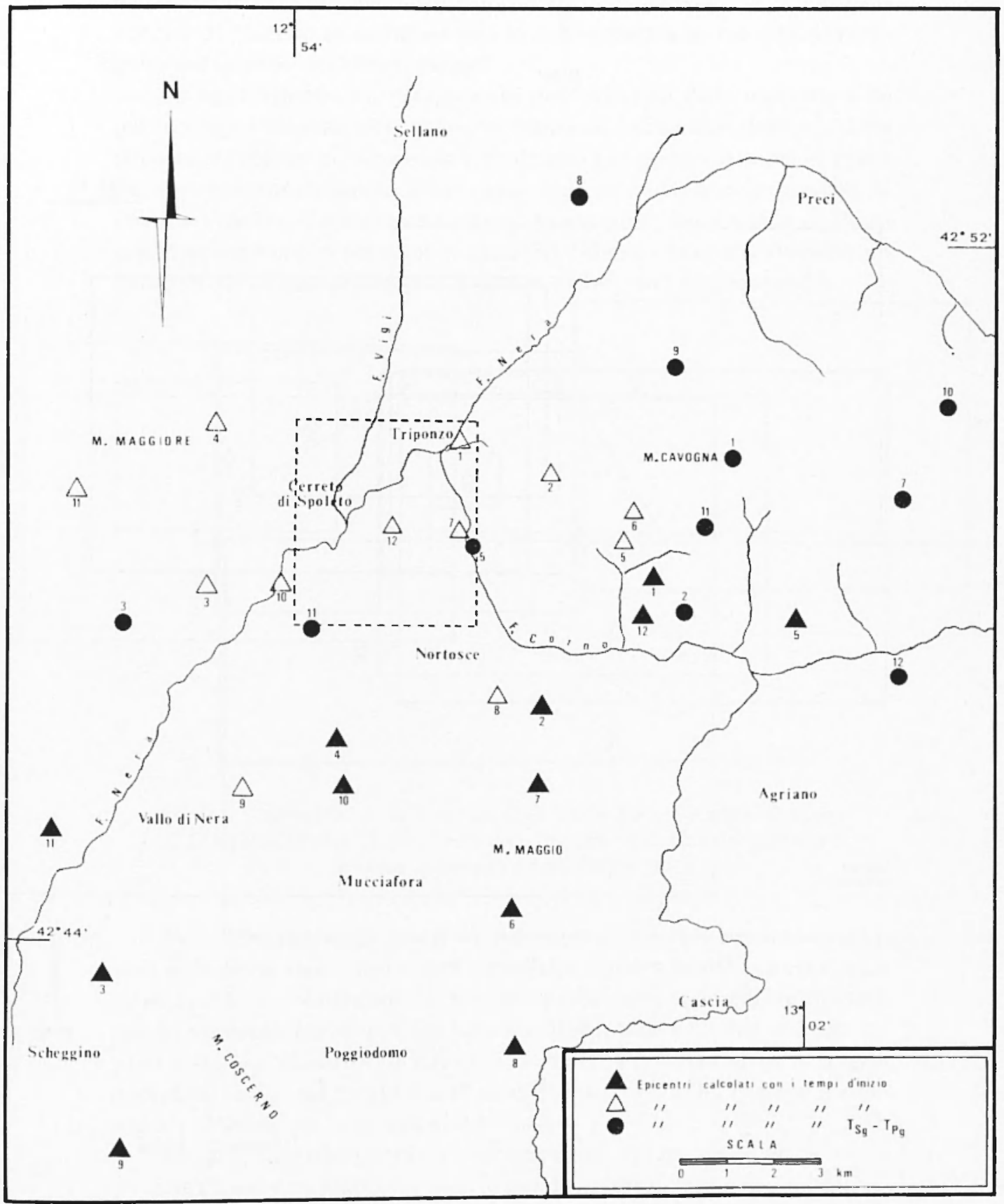

Fig. 4 - Epicentri determinati con i metodi deseritti nel testo. La numerazione segue l'ordine cronologieo degli oventi. La parte trattegrgiata si riferisco allarea coperta dalla fig. e. 

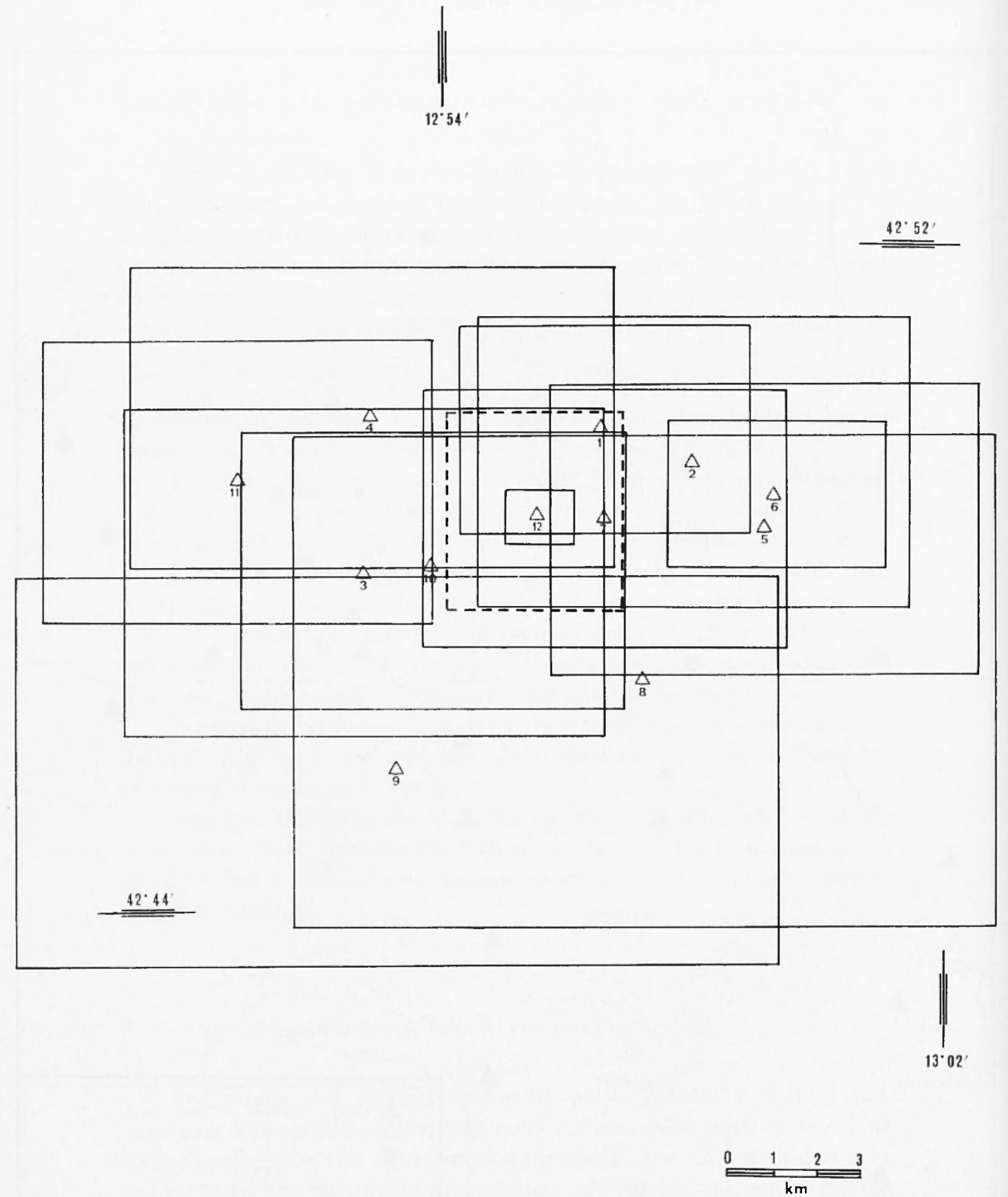

Fig. 5 - l'osizioni degli epicentri di fig. 4 (triangoli hianchi) con i relativi errori standard. Si può notare che i soli epicentri 6 e 11 non si sovrappongono alla zona epicentrale (parte trattegrgiata). 
metri di partenza. La coerenza con cui in ogni stazione si sono misurati i tempi di tragitto fa eschudere che le differenze fra le velociti provengano solamente da errori asuali.

In fig. 6 riportiamo l'andamento delle velocità delle onde $P g$ e śg per le cinque stazioni più vicine, in funzione dell'azimut della stazione rispetto all'epicentro, ritenendo che almeno per queste stazioni si possa parlare inconfutabilmente dello stesso tipo di onda. Per le stazioni di Prato e Firenze, che hamno un azimut molto prossimo a quello di Perusria, riteniamo che le prime onde giuntevi debbano aver attraversato un paceo di strati più profondo e veloce e quindi non confrontabile.

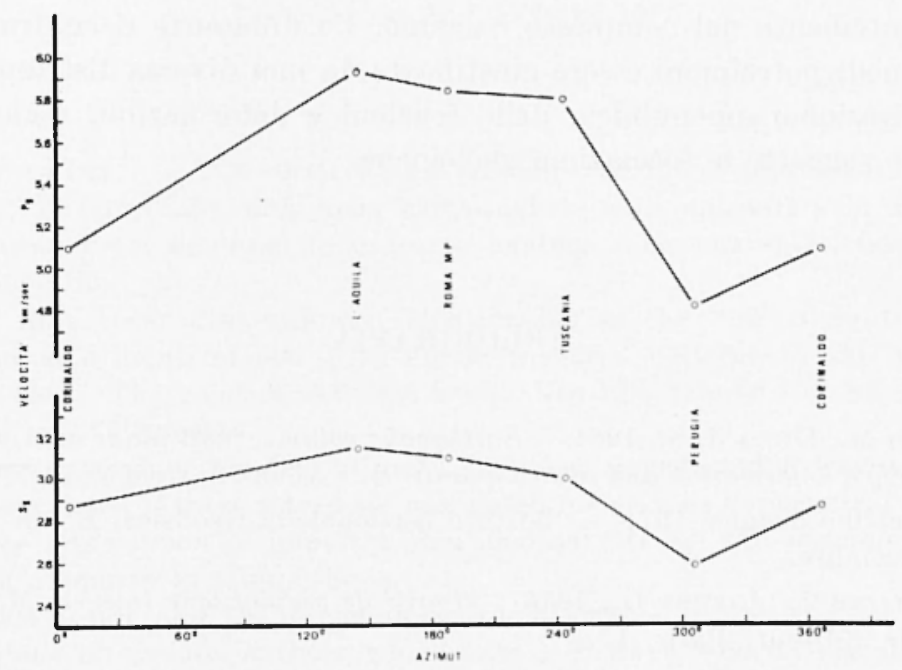

Firg. 6 - Variazioni della velocita delle onde Pg e Sg nelle stazioni di L'Aquila, Roma M.P., Tuscania, Perugia e Cormaldo installate intorno a Cerreto come da fig. 1.

Le profondità degli ipocentri collocherebbero il movimento tettonico all'interno delle formazioni triassiche o pre-triassiche umbre, cioc oltre quella che costituisce la base affiorante della serie umbro-marchigiana. Le rocee costituenti la formazione di base nellarea epicentrale sono date da calcari subcristallini e clolomie; sottostante a queste lovrebbe esserci un complesso di tipo filladico, o simile, che per convenienza chiameremo "complesso di base".

Le onde sismiche debbono aver effettuato il loro massimo percorso all'interno del complesso di base, o per lo meno in roce con caratteristiche meccaniche grosso modo analoghe. Per i tragitti Cerreto-Tusca- 
nia e Celreto-Roma M.P., la velociti delle onde $P g$ si può considerare costante (5.81 e $5.85 \mathrm{~km} / \mathrm{sec}$ ). Il complesso di base dovrebbe avere una distribuzione quasi omogenea e le anomalie gravimetriche confermerebbero tale ipotesi. L'andamento del complesso di base non dovrebbe essere molto differente dal precedente anche per il percorso CerretoPerugia, dove invece si riscontra la velocità più bassa, $4.82 \mathrm{~km} / \mathrm{sec}$.

Verso Nord, percorso Cerreto-Corinaldo (velocità $5.09 \mathrm{~km} / \mathrm{sec}$ ), il complesso di base si troverebbe a profondità maggiore, per cui le onde avrebbero effettuato un percorso misto tipo complesso base-complesso calcareo, il che giustificherebbe una diminuzione di velocità. Una velocità ancora minore dovrebbe riscontrarsi nel percorso Cerreto-L'Aquila, prevalentemente nel complesso calcareo. Le differenze riscontrate dai valori medi potrebbero essere giustificate da una cliversa distribuzione, nella direzione appenninica, delle tensioni e deformazioni regionali d cui sono soggette le formazioni geologiche.

\section{BIBIAOGRAFIA}

(1) BÅT1 M., 1)UDA .I. S., 1964. - Earlhquale volume, faull plane area, seismic energy, deformation and relaled quantilies. "Annali di Geofisica "XVIII, 3

(2) Bollettino Sismico 1974. - "Istituto Nazionale di Geofisica, Roma M.P. ", Dicembre.

(3) Coulous .J., Jobert G., 1973. - Traité de géophysique inlerne. Massen e ('ie Editeur, Paris, I.

(4) Di Fidippo 1)., Marceldi L., 1952. - Dromocrone per lerremoli vicini e velociti delle onde nell Italia Centrale. "Annali di Geofisica ", V, 2.

(5) L'rsu T., SЕк1 A., 1955. - Relation between the area of the aftershock region and the energy of the main shock. "Journ. Seism. Soc, "Japan, serie II. VII. 